\title{
Dimensional Reduction in Classical Lorenz System
}

\author{
P. MIŠKINIS* \\ Vilnius Gediminas Technical University, Sauletekio al. 11, 10223 Vilnius, Lithuania
}

Received: 29.11.2020 \& Accepted: 19.02.2021

Doi: $10.12693 /$ APhysPolA.139.649

*e-mail: paulius.miskinis@vilniustech.lt

\begin{abstract}
Important properties of dynamical systems with a nonlocal evolution operator in the form of CaputoWeyl are considered. A double dimensional reduction of the evolutionary operator of a special form connects various nonlinear fractional dynamical systems. For the integer values of the fractional parameter, we obtain the interrelation among various classical dynamical systems. In particular, it is shown that with a special choice of the evolution operator and dimensional reduction, the one-dimensional evolutionary Richards equation, the two-dimensional Gierer-Meinhardt system and the classical Lorenz system are closely interconnected dynamical systems.
\end{abstract}

topics: nonlinear and nonlocal theories and models, fractional calculus, nonlinear dynamics

\section{Introduction}

In the recent decade, in connection with diverse problems of natural science, various methods of the fractional integral and differential calculus have become increasingly popular.

The dynamical systems (DS) theory is one of the most powerful and far-advanced tools of modern theoretical physics $[1,2]$. With the development of science, not only did the methods improve, but the very concept of DS changed. Currently, the theory of DS is a collective name for a wide range of studies, where various methods of modern mathematics and theoretical physics are used and fruitfully combined.

The use of methods of fractional analysis gave rise to a new concept - fractional dynamical systems (FDS). Among researchers working in this area, there is a growing understanding that FDS is not only a new powerful tool for modern theoretical and applied research, but also a deep and far-reaching generalization of the concepts of classical DS [3]. Just as in the case of the classical $\mathrm{DS}$, in the most general case, discrete and continuous FDS are distinguished [4, 5]. In discrete FDS or fractional cascades, the set of possible moments in time forms a discrete set, and the behavior of the system is described by a sequence of discrete states. A sequence of discrete states in the phase space of the FDS forms the trajectory of the cascade.

In continuous FDS or continuous flows, the set of possible points in time forms a continuous set, and the behavior of the FDS is described by a sequence of continuous states. The set of possible points in time and, accordingly, the state of the flow as a rule are determined for each point of the real or complex axis.

It is well known that cascades and flows of the classical DS are the main subject of consideration in symbolic and topological dynamics [6] and are in demand in other branches of natural science, such as nonequilibrium thermodynamics [7, 8], the theory of dynamic chaos [9] and synergetics [2].

The main mathematical tool of the FDSs is a method of the fractional differential equations. The fractional differential equations and the related aspects were considered by many authors, e.g., $[10,11]$.

In particular, to describe the properties of flows of an FDS, it is necessary not only to generalize the definition of the evolution operator, but also of the group of diffeomorphisms [3, 15, 16]. The complex form of the evolution operator, even for relatively simple FDS flows, leads to the fact that numerical solution methods become the main tool. The few known analytical solutions are rare exceptions [12-14].

This paper announces an important property of the FDS: a continuous change in the fractional parameter of the evolutionary operator connects various nonlinear fractional DSs and, for integer values of the parameter, the classical ones. As an example, a continuous change in the fractional parameter in the classical nonlinear Lorenz system is traced. In particular, it is shown that, under a special choice of the evolution operator and dimensional reduction, 
the one-dimensional evolutionary Richards, the two-dimensional Gierer-Meinhardt and the threedimensional classical Lorenz systems are closely interconnected DSs.

\section{Fractional Lorenz system}

Let the derivative's order of the FDS change from the fixed value to zero. At the $\alpha_{i}=0$, the corresponding equation of motion $(i)$ turns into a constraint and changes the evolution of the whole dynamical system.

As an example, let us consider the classical Lorenz system (see [17]), where $(x, y, z) \in \mathbb{R}^{3}$,

$$
\left\{\begin{array}{l}
\dot{x}=\sigma(y-x), \\
\dot{y}=\rho x-y-x z, \\
\dot{z}=x y-\beta z .
\end{array}\right.
$$

All parameters of the Lorenz system are positive $\sigma, \rho, \beta>0$ and have a specific physical content. Namely, $\sigma$ is the Prandtl number, $\rho$ is the reduced Rayleigh number, and $\beta$ is the geometrical ratio of the model. The classical values of the parameters used by Lorenz are as follows: $\sigma=10, \beta=8 / 3$ and $\rho=28$ [19]. The Lorenz system was historically the first meaningful example of the ODE system with a strange attractor which had explicit physical applications.

Let us consider the fractional generalization of the classical Lorenz system, where we have to substitute the time derivative by the evolutionary operator of the fractional derivative in the form of Caputo-Weyl ${ }_{-\infty} D_{t+}^{\alpha}$. Then, we deal with

$$
\begin{aligned}
& \frac{\mathrm{d}}{\mathrm{d} t} \rightarrow \frac{\mathrm{d}^{\alpha}}{\mathrm{d} t^{\alpha}}={ }_{-\infty} D_{t+}^{\alpha}-\delta_{\alpha, 0}, \\
&-\infty D_{t+}^{\alpha} f(t)= \\
& \frac{1}{\Gamma(n-\alpha)} \int_{-\infty}^{t} \frac{\mathrm{d} \tau}{(t-\tau)^{1+\alpha-n}} \frac{\mathrm{d}^{n} f(\tau)}{\mathrm{d} \tau^{n}},
\end{aligned}
$$

where $\delta_{\alpha, 0}$ is the Kronecker delta with $\alpha>0$, $\Gamma(x)$ is the Euler gamma function, $n$ is expressed as $n=\lfloor\alpha\rfloor+1$ and $\lfloor\alpha\rfloor$ means the integer part of the order $\alpha$.

Thus, we have not the classical but the fractional Lorenz system (FLS):

$$
\left\{\begin{array}{l}
\dot{x}^{\left(\alpha_{x}\right)}=\sigma(y-x), \\
\dot{y}^{\left(\alpha_{y}\right)}=\rho x-y-x z, \\
\dot{z}^{\left(\alpha_{z}\right)}=x y-\beta z,
\end{array}\right.
$$

where $0 \leq \alpha_{i} \leq 1,(x, y, z) \in \mathbb{R}^{3}$. For each set of the derivative orders $\left(\alpha_{x}, \alpha_{y}, \alpha_{z}\right)$, we have different FLS.

There are a few examples in the literature of the fractional generalization of the LS [20-22] (and references therein). We, however, are interested in the case with different derivative orders $\left(\alpha_{x}, \alpha_{y}, \alpha_{z}\right)$ and the evolutionary time operator of $(2)$, which has not yet been considered. This allows us to apply the dimensional reduction and some interesting relations.
The FLS equation (4) is invariant under transformations $(x, y, z) \rightarrow(-x,-y, z)$ and has three fixed points, i.e., the origin of the coordinates $O=(0,0,0)$ and two points $O_{1,2}$

$$
O_{1,2}=( \pm \sqrt{\beta(\rho-1)}, \pm \sqrt{\beta(\rho-1)}, \rho-1) .
$$

Due to the Kronecker symbol $\delta_{\alpha, 0}$ in the evolutionary operator (2), the fixed points of the FLS remain unshifted for $\alpha=0$. Their stability depends on the values of the parameters.

\section{Dimensional reduction of fractional Lorenz system}

A qualitative difference in the properties of a dynamical system (DS) of the integer and fractional order has always been noted (see the monographs $[3,23])$. Even a small deviation of the order of the differential operator from an integer value $\alpha \rightarrow n-\varepsilon$ means the transformation of the DS into an FDS, i.e., a non-conservative DS. This, in turn, means the disappearance of one or several integrals of motion, a change of the asymptotic states, and a qualitative change in the solution as a whole.

Unfortunately, not enough attention is paid to the study of cases when the order of the differential operator $\alpha$ changes significantly, i.e., $\alpha \rightarrow n-1+\varepsilon$. In this case, we can talk about the reduction or degeneration of the DS into the DS of a lower order. The idea of interrelation among DSs of different orders is not new (see the monograph [24]), however, in fractional DSs it is practically not studied. A continuous change of the order of an FDS makes it possible to trace this process in detail.

Let us apply the two-dimensional reduction to the FLS when only one dynamical variable remains unchanged. In the three-dimensional case, it can be done independently in three ways: $\quad$ (i) $(x) \rightarrow(y) \rightarrow(z)$; (ii) $(x) \rightarrow(y) \leftarrow(z)$; and (iii) $(x) \leftarrow(y) \leftarrow(z)$. With the used notation, the scheme, for example, $(x) \rightarrow(y) \rightarrow(z)$, means that the equation for the function $x(t)$ at $\alpha_{x} \rightarrow 0$ becomes a constraint and it is substituted into the equation for the function $y(t)$. Further, at $\alpha_{y} \rightarrow 0$, the equation for the function $y(t)$ becomes a new constraint and is substituted into the equation for the function $z(t)$. Note that after the first substitution we have a two-dimensional system. After the second substitution, in turn, we obtain a onedimensional differential equation of the first order.

Analogously, one should read the scheme $(x) \rightarrow(y) \rightarrow(z)$. It means that the equation for the function $x(t)$ at $\alpha_{x} \rightarrow 0$ becomes a constraint and is substituted into the equation for the function $y(t)$. At $\alpha_{y} \rightarrow 0$, the equation for the function $y(t)$ becomes a new constraint and is substituted into the equation for the function $z(t)$. After the first substitution, we have a two-dimensional system, and after the second substitution we obtain a one-dimensional differential equation of the first order. Therefore, let us consider all the three cases in the following subsections. 


$$
\text { 3.1. DR: }(x) \rightarrow(y) \rightarrow(z)
$$

The first two equations in (2) turn into two constraints. Upon substituting them into the third equation of motion, we should obtain a onedimensional dynamical system. However, in this case the double DR does not give us any dynamical equation and degenerates into a set of the same fixed points $O, O_{1}$ and $O_{2}$ in (5).

$$
\text { 3.2. DR: }(x) \rightarrow(y) \leftarrow(z)
$$

The FLS turns into the system

$$
\left\{\begin{array}{l}
x=y, \\
\dot{y}^{\left(\alpha_{y}\right)}=(\rho-1)\left(1-\frac{y^{2}}{\beta}\right) y, \\
z=\frac{y^{2}}{\beta} .
\end{array}\right.
$$

For $\alpha_{y}=1$, the second equation in (6) is the Richards differential equation [25] - the evolutionary equation well known in biology:

$$
\dot{y}=\left(1-\left(\frac{y}{K}\right)^{\nu}\right) \text { ay }
$$

with $\nu>0, a>0$. The solution of this equation is the generalized logistic curve [26]:

$$
\begin{aligned}
& y(t)=K\left(1+Q \mathrm{e}^{-a \nu\left(t-t_{0}\right)}\right)^{-1 / \nu}, \\
& Q=\left(\frac{K}{y_{0}}\right)^{\nu}-1, \quad y_{0}=y\left(t_{0}\right),
\end{aligned}
$$

which, alongside the ordinary logistic curve $(\nu=1)$, is a function widely used for modeling the population growth. Therefore, the solution of the second equation of the system for $\alpha_{y}=1$ is

$$
y(t)=\sqrt{\frac{\beta}{1+\left(\beta / y_{0}^{2}-1\right) \mathrm{e}^{-2(\rho-1)\left(t-t_{0}\right)}}}
$$

and $y_{0}=y\left(t_{0}\right)$.

Figure 1 shows the classical three-dimensional Lorenz system. As an example, in Figs. 2 and 3, asymptotic states of the dimensional reduction of a fractional Lorenz system in case (b) are

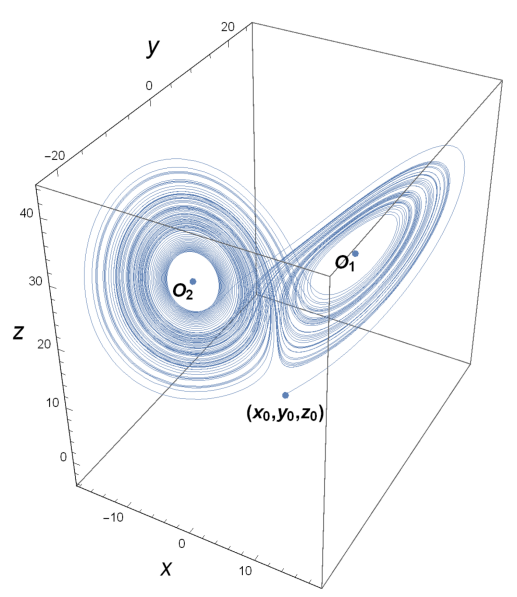

Fig. 1. Classical three-dimensional Lorenz system (1). The values of the parameters are classical: $\sigma=10, \beta=8 / 3$ and $\rho=28$.

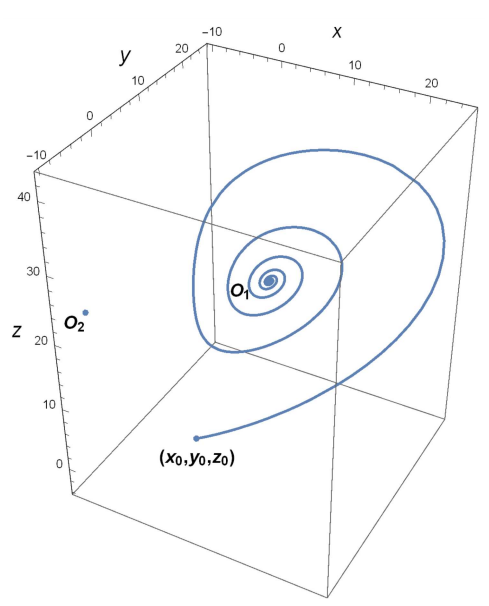

Fig. 2. The Lorenz system (1) after a onedimensional reduction. The value of the parameters, fixed points and initial conditions are the same.

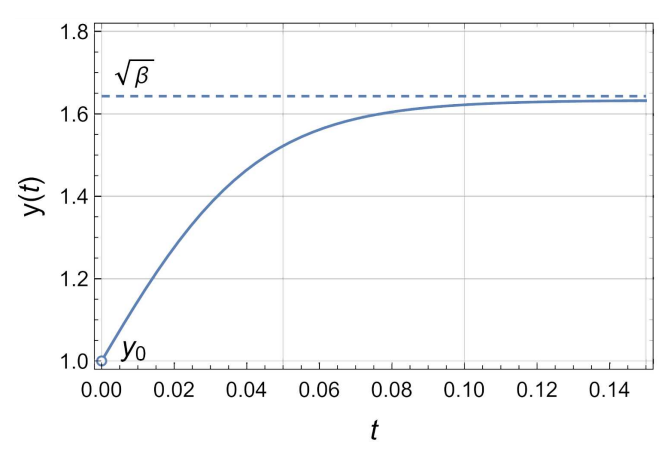

Fig. 3. Solution of (9) and the asymptotic state $y_{a s}=\sqrt{\beta}$ for $t \rightarrow \infty$. The behavior of this onedimensional system depends only on the two initial parameters $\rho$ and $\beta$.

presented. The values of the dimensionless parameters are the same as in the classical case (1). The fixed points $O_{1}$ and $O_{2}$ in (5), corresponding to these parameters, and the initial condition chosen in the form $x_{0}=y_{0}=z_{0}=1$ are marked in the figure.

After the dimensional reduction $(x) \rightarrow(y)$, the first equation becomes a constraint, and the system effectively becomes two-dimensional. The result of this reduction is shown in Fig. 2. Note that due to the special choice of the differentiation operator (2), the position of the fixed points of the reduced system does not change. The system becomes independent on the parameter $\sigma$. In the special case of the Richards equation, for $\nu=1$, the logistic equation corresponds to the initial Rössler-type system [27].

After the second dimensional reduction of $(z) \rightarrow(y)$, the third equation becomes a constraint, and the system effectively becomes onedimensional. Figure 3 shows the solution of (9) and the asymptotic state $y_{a s}=\sqrt{\beta}$ for $t \rightarrow \infty$. The system depends on two initial parameters: $\rho$ and $\beta$. 


$$
\text { 3.3. DR: }(x) \leftarrow(y) \leftarrow(z)
$$

The FLS turns into the system

$$
\left\{\begin{array}{l}
\dot{x}^{\left(\alpha_{x}\right)}=\left(\frac{\rho}{1+x^{2} / \beta}-1\right) \sigma x \\
y=\frac{\rho x}{1+x^{2} / \beta} \\
z=\frac{x y}{\beta} .
\end{array}\right.
$$

assuming $x_{a s}=\sqrt{(\rho-1) \beta}, \quad y_{a s}=x_{a s}$, and $z_{a s}=\rho-1$. On the right-hand side of the system (10), its fixed points at $t \rightarrow \infty$ are indicated. For $\alpha_{x}=1$, the first equation of the system (10) is related to the Gierer-Meinhardt model well known in biology [28], which in the dimensionless form could be expressed as

$$
\left\{\begin{array}{l}
\dot{x}=a-B x+\frac{x^{2}}{y\left(1+K x^{2}\right)}, \\
\dot{y}=x^{2}-y
\end{array}\right.
$$

where $a, B$ and $K$ are the constants. The model described by (11) also has an important application in chemistry, where it describes a two-component autocatalytic reaction. The term $\frac{x^{2}}{y\left(1+K x^{2}\right)}$ corresponds to the production of the activator $x$. For $x \gg 1$, the quantity of the activator $x$ achieves the saturation value $1 /(K y)$. The inhibitor production $y$ is activated by the activator $x$ and corresponds to the second equation of the equation system (11).

The first equation of the one-dimensional GiererMeinhardt model for $y=x$ and $a=0$ directly leads to the first equation in (10) for $\alpha_{x}=1$ :

$$
\dot{x}=\frac{x}{1+K x^{2}}-B x .
$$

The correspondence of the parameters in (10) and (12) could be expressed in the explicit form $\left(\alpha_{x}=1\right)$ :

$$
\sigma=B, \quad \beta=1 / K, \quad \rho=1 / B
$$

The model (12) is used in ecology as a model of population dynamics with the polynomial growth and constant trade of the population [29]. This model and the result of the dimensional reduction (10) admit the analytical solution with the dependence of the model parameters. The analytical solution of

$$
\dot{x}=\left(\frac{\rho}{1+x^{2} / \beta}-1\right) \sigma x,
$$

when $x(0)=x_{0}$, is an analytical function in the unexplicit form:

$$
e^{2 b t}=\frac{1}{\xi^{2}}\left(\frac{\xi^{2}+a}{1+a}\right)^{\rho} .
$$

Here

$$
\begin{aligned}
& a \equiv(1-\rho) \beta / x_{0}^{2}, \quad b \equiv(1-\rho) \sigma \\
& \xi \equiv x / x_{0} .
\end{aligned}
$$

The analytical solution of (10) is shown in Fig. 4. For better visibility, the scale of the plots has been changed.

Thus, we may conclude that after the twodimensional reduction, the initial FLS (4), depending on the way of reduction, reduces to the Richards or a special case of the Gierer-Meinhardt model or the model of the population dynamics.

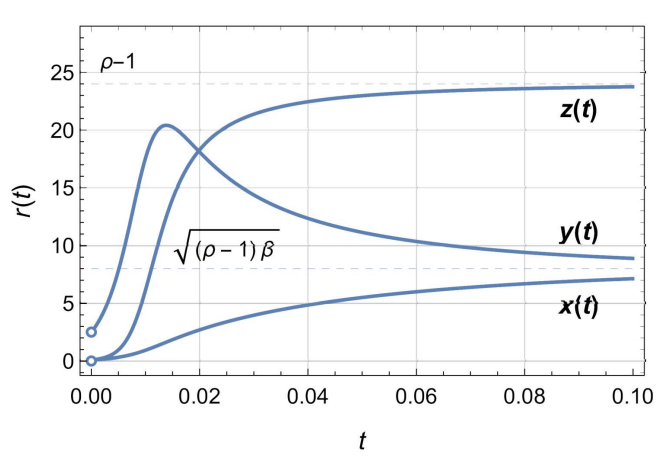

Fig. 4. Solutions of the system (9) after a twodimensional reduction and their asymptotic states $x_{a s}=y_{a s}=\sqrt{(\rho-1) \beta}$ and $z_{a s}=\rho-1$. The behavior of this degenerate system also effectively depends only on the two initial parameters.

Note here that the differential operator (2) continuously depends on the derivative order $\alpha$. This means that by changing the derivative order we obtain a continuous transition from one FLS to another. Nevertheless, this deformation of the phase flow is certainly not a topological map, because the dimension of the phase flow is a topological invariant.

It is no wonder that, upon performing certain procedures, a three-dimensional DS turns into a onedimensional DS. The point, however, is that this type of dimensional reduction allows answering the question which is of particular significance in the theory of dynamical systems: how in a complex onedimensional DS is it possible to see a simple multidimensional DS? The example of the FLS shows that the three-dimensional Lorenz system may be restored from the one-dimensional Richards equation (7) (with the accuracy up to the $\sigma$-parameter) and from the one-dimensional Gierer-Meinhardt equation (12).

\section{Physical aspects of fractional Lorenz system}

The simplest fractional physical systems, for example, the fractional harmonic oscillator [3, 4], show that such systems are physical systems with dissipation. Since the real physical systems observed in nature are most often dissipative, this means that FLS is more realistic than the classical LS. In this sense, the classical LS can be considered as a special case of FLS.

Edward Lorenz proposed his LS (1) as a simplified mathematical model of atmospheric convection [17-19]. Originally, LS is a three-dimensional physical system. The method of dimensional reduction proposed in the article shows how a three-dimensional physical LS (1) can degenerate into a two- and even one-dimensional system (see (6) and (10)).

The continuous change in the order of the system $\alpha_{i}$ allows us to trace in detail theprocess 
of degeneration of a physical system from threedimensional to one-dimensional. Due to the relative simplicity of the nonlinear LS, the solutions of the corresponding reduced evolutionary equations can be represented in an analytical form (see (1) and (14)-(16)).

Dimensionless parameters of the model $\sigma, \rho$ and $\beta$ have a direct physical meaning. Therefore, $\sigma$ is the Prandtl number $\operatorname{Pr}, \rho$ is the normalized Rayleigh number $\mathrm{Ra}$, and $\beta$ is the geometric parameter of the model. The dimensional reduction of the LS shows how the corresponding parameters of the model are interrelated. As follows from the solutions (6) and (10), in this scheme of dimensional reduction, $(\rho-1)$ determines the rate of the two-dimensional evolutionary process, and $\sqrt{\beta}$ is the limiting maximum value of the dimensionless quantity $y$. The physical meaning of the quantity $y$ depends on the specific type of the model.

It is well known that the Lorenz equations also arise in simplified models of laser physics, in some models of dynamos, thermosiphons, brushless DC motors, electrical circuits, chemical reactions, and direct osmosis. The Lorenz equations are also the governing equations in the Fourier space for the Malkus water wheel (see $[17,18]$ and references therein).

In the classical LS and the models listed above, the effective degeneration of a three-dimensional system into a two- or even one-dimensional system is quite appropriate and is of special interest. In all cases which are reduced to the Lorenz model, the results of this article are applicable, demonstrating the relationship of the corresponding solutions and model parameters. It is interesting that the proposed dimensional reduction is applicable in the opposite direction: from the one-dimensional system (10), by means of inverse transformations, one can obtain a two-dimensional system (6) and a three-dimensional system (1). This means that if we know that the one-dimensional approximation of a physical process evolves according to the model (10), and we know that the system is actually three-dimensional, then, according to the proposed method, it can be completely restored. So, in the case of a one-dimensional logistic model, the dimensional reduction connects this model to another well-known nonlinear model, the so-called Rössler model [27].

The emergence of a related nonlinear model suggests the idea of applying the proposed method of dimensional reduction to other nonlinear evolutionary systems. The application of the proposed technique in the theory of the second-order phase transitions also looks promising.

\section{Conclusions and discussion}

As follows from the two-dimensional reduction, the nonlinear Lorenz system relates to the Richards and the Gierer-Meinhardt systems and depends on the type of reduction.
From the mathematical point of view, a sufficient condition for the existence of a fractional derivative belongs to the class of continuous functions, see [13]. In other words, a continuous but non-differentiable function can never be the solution of a classical DS, while it can be for an FDS. The physical content of the FDS is in the process of construction [3, 23], but even now we can note that FDSs are a generalization of the classical DSs when the classical concept of velocity does not work.

The core object is the evolutionary operator in the form of the fractional derivative. However, the latter may be not only in the Caputo-Weyl form, but also in that of Riemann-Liouville, Riesz, Feller, Hadamard etc., see [30]. How much do the FDS properties depend on the form of its introduction? The basic criterion while introducing the FDS is the physical correspondence between the fractional and the classical DSs systems: when the order of the fractional derivative $\alpha \rightarrow 1$, an FDS must turn into a classical DS: $F D S \underset{\alpha \rightarrow 1}{\stackrel{D}{\longrightarrow}} S$.

Another important field of FDS is the dimensional reduction: for $\alpha \rightarrow 0$, part of the dynamical equations turn into constraints. We obtain a unique tool: the phase semi-flow of the rank $n$ under continuous limit transition $\alpha \rightarrow 0$ turns into a phase semi-flow of the rank $n-1$ and into certain constraints. An interesting result concerning the classical Lorenz system has been considered in Sect. 3 . In the general case, the correlation among the classical DSs of different dimensions deserves a detailed analysis.

The above-considered FDSs belong to the class of continuous DSs. The relation of the FDS with the ergodic theory and topological DSs is absolutely unclear.

\section{Acknowledgments}

The author would like to express his gratitude to Professor M. Pavlov for the invitation and the possibility to contribute to an international mathematical conference at the Moscow State University and for numerous discussions with colleagues.

\section{References}

[1] Contemporary Approaches and Methods in Fundamental Mathematics and Mechanics, Eds. V.A. Sadovnichiy, M.Z. Zgurovsky, Understanding Complex Systems Series, Springer, 2020.

[2] Synergetics, Eds. A. Hutt, H. Haken, Encyclopedia of Complexity and Systems Science Series, Springer, 2020.

[3] Handbook of Fractional Calculus with Applications, Vol. 4 - Applications in Physics, Part A, Ed. V.E. Tarasov, De Gruyter, New York 2019. 
[4] Fractional Calculus and Fractional Differential Equations, Ed. V. Daftardar-Gejji, Birkhäuser Basel, 2019.

[5] C. Goodrich, A.C. Peterson, Discrete Fractional Calculus, Springer International Publishing, Cham (Switzerland) 2015.

[6] J. Vries, Topological Dynamical Systems: An Introduction to the Dynamics of Continuous Mappings in series De Gruyter Studies in Mathematics, Book 59, De Gruyter, Berlin 2014.

[7] S. Kjelstrup, D. Bedeaux, Non-Equilibrium Thermodynamics of Heterogeneous Systems in series Advances in Statistical Mechanics, Vol. 16, World Scientific Publishing Company, 2008.

[8] E. Barsky, Entropy of Complex Processes and Systems Elsevier, 2020.

[9] Analysis, Probability and Mathematical Physics on Fractals, Eds. P.A. Ruiz, J.P. Chen, L.G. Rogers, R.S. Strichartz, A. Teplyaev, in series Fractals and Dynamics in Mathematics, Science, and the Arts: Theory and Applications, Book 5, World Scientific Publishing Company, 2020.

[10] S.G. Georgiev, Fractional Dynamic Calculus and Fractional Dynamic Equations on Time Scales Springer, 2018.

[11] C. Milici, G. Drăgănescu, J.T. Machado, Introduction to Fractional Differential Equations, in series Nonlinear Systems and Complexity, Book 25, Springer, 2018.

[12] Handbook of Fractional Calculus with Applications, Vol. 1: Basic Theory, Eds. A. Kochubei, Y. Luchko, in series De Gruyter Reference, De Gruyter, 2019.

[13] Handbook of Fractional Calculus with Applications, Vol. 2: Fractional Differential Equations, Eds. A. Kochubei, Y. Luchko, in series De Gruyter Reference, De Gruyter, 2019.

[14] Handbook of Fractional Calculus with Applications, Vol. 3: Numerical Methods, Ed. G.E. Karniadakis, in series De Gruyter Reference, De Gruyter, 2019.
[15] P. Miškinis, Math. Model. Anal. 15, 235 (2010).

[16] P. Miškinis, Nonlinear \& Nonlocal Integrable Models Technika, Vilnius 2003.

[17] J. Guckenheimer, P. Holmes, Nonlinear Oscillations, Dynamical Systems, and Bifurcations of Vector Fields, SpringerVerlag, New York 1983.

[18] C. Sparrow, The Lorenz Equations: Bifurcations, Chaos, and Strange Attractors, Springer-Verlag, New York 1982.

[19] E.N. Lorenz, J. Atmos. Sci. 20, 130 (1963).

[20] R.W. Ibrahim, Abstr. Appl. Anal. 2013 127103 (2013).

[21] I. Grigorenko, E. Grigorenko, Phys. Rev. Lett. 91, 034101 (2003).

[22] K. Sun, J.C. Sprott, Electron. J. Theor. Phys. 6, 123 (2009).

[23] Handbook of Fractional Calculus with Applications, Ed. V.E. Tarasov, Vol. 5: Applications in Physics, Part B, in series De Gruyter Reference, De Gruyter, 2019.

[24] A.N. Tikhonov, A.A. Samarskiy, Equations of Mathematical Physics, Dover Books on Physics, New York 2011.

[25] F.J. Richards, J. Exp. Bot. 10, 290 (1959).

[26] Y.C. Lei, S.Y. Zhang, Nonlinear AnalModel. 9, 65 (2004).

[27] O.E. Rössler, Phys. Lett. A 71, 155 (1979).

[28] H. Meinhardt, Models of Biological Pattern Formation, Academic Press, London, 1982.

[29] M. Begon, C.R. Townsend, J.L. Harper, Ecology: From Individuals to Ecosystems, Blackweel, Malden (MA) 2006.

[30] X-J. Yang, F. Gao, J. Yang, General Fractional Derivatives with Applications in Viscoelasticity, Academic Press, London 2020. 